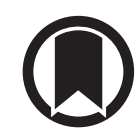

CrossMark

\section{Genetically proxied interleukin-6 receptor inhibition: opposing associations with COVID-19 and pneumonia}

\author{
To the Editor:
}

The inflammatory cytokine interleukin-6 (IL-6) is central to orchestrating the immune system [1]. The pathophysiological process underlying severe coronavirus disease 2019 (COVID-19), caused by the severe acute respiratory syndrome coronavirus 2 , consists of an exaggerated host immune response and elevated circulating levels of inflammatory cytokines, including IL-6 $[2,3]$. As such, immunomodulatory agents are being investigated for the treatment of COVID-19. Glucocorticoids may limit inflammation-mediated lung injury in patients with severe COVID-19, and consequently reduce progression to respiratory failure and death. The RECOVERY trial found that administration of dexamethasone resulted in lower 28-day mortality among hospitalised COVID-19 patients who were receiving either invasive mechanical ventilation or oxygen alone at randomisation, but not among those who were not receiving any respiratory support [4]. IL-6 receptor (IL6R) inhibition may represent another potential immunomodulatory strategy for treating COVID-19 [5, 6], and a recent meta-analysis of mean IL-6 concentrations demonstrated 2.9-fold higher levels in patients with complicated COVID-19 compared with patients with non-complicated disease [7].

Genetic variants that proxy IL6R inhibition may be used as instrumental variables in the Mendelian randomisation paradigm to investigate corresponding drug effects. Here, we conducted a Mendelian randomisation investigation to assess the potential effect of IL6R inhibition on COVID-19 susceptibility and hospitalisation and risk of pneumonia.

IL6R inhibition was proxied using seven single-nucleotide polymorphisms within or adjacent to the IL6R locus that were associated with C-reactive protein (CRP; downstream molecule of IL-6 signalling) concentrations at the genome-wide significance threshold in 204402 individuals of European ancestry [8]. These genetic variants also had associations with fibrinogen, IL-6 and soluble IL-6 receptor in a pattern consistent with their effect on IL6R inhibition [8]. The variance in CRP concentrations explained by each variant ranged from $0.04 \%$ to $0.34 \%$.

Summary-level genetic data for COVID-19 were acquired from: 1) The COVID-19 Host Genetics Initiative genome-wide association meta-analysis (release 4, 20 October 2020; without the 23andMe study) [9], which included 17965 COVID-19 cases versus 1370547 population controls, 7885 hospitalised COVID-19 cases versus 961804 population controls, and 4336 very severe respiratory confirmed COVID-19 cases versus 623902 population controls; and 2) a genome-wide association study involving 1610 hospitalised patients with severe COVID-19, defined as having respiratory failure and requiring some degree of respiratory support, and 2205 control participants (healthy volunteers, blood donors and outpatients of gastroenterology departments) from seven hospitals in Italy and Spain [10]. Corresponding data for pneumonia were obtained from: 1) the FinnGen consortium, including 15778 cases of all pneumoniae (International Statistical Classification of Diseases and Related Health Problems - 10th Revision codes: J12-J16, J18), and 119867 control participants [11]; and 2) the UK Biobank cohort (through the MRC-IEU consortium via the MR-Base platform [12]), which included 6572 self-reported pneumonia cases (id:UKB-b:4533) and 456361 control participants. The genetic association estimates in all studies

@ERSpublications

Respiratory disease is a main feature of severe COVID-19, and the potential of IL-6 receptor blockade to increase risk of pneumonia warrants vigilance and caution in its application to treat COVID-19 https://bit.ly/34Y8Ner

Cite this article as: Larsson SC, Burgess S, Gill D. Genetically proxied interleukin-6 receptor inhibition: opposing associations with COVID-19 and pneumonia. Eur Respir J 2021; 57: 2003545 [https://doi.org/ $10.1183 / 13993003.03545-2020]$. 
were adjusted for sex and ancestry. Only summary-level data were analysed in this study, for which appropriate ethical approval and participant consent had previously been obtained.

The Mendelian randomisation analyses were performed using the multiplicative random-effects inverse-variance weighted method and accounting for correlations between genetic variants. Analyses were conducted in $\mathrm{R}$ (version 3.4.3) using the MendelianRandomization package [13]. Our results showed that genetically proxied IL6R inhibition, scaled per 0.1 standard deviation decrease of natural log-transformed CRP concentrations, was associated with a reduced risk of being infected and hospitalised with COVID-19, but also with an increased risk of pneumonia (figure 1).

Our findings provide evidence supporting that IL6R inhibition reduces the risk of being infected and hospitalised with COVID-19. Importantly, they also go further to provide genetic evidence that IL6R inhibition may increase susceptibility to pneumonia. While we considered a broad definition of pneumonia from any cause, our results may be of relevance to secondary lung infections that can complicate COVID-19. The neutral finding of a recent randomised controlled trial of IL6R blockade for the treatment of hospitalised COVID-19 [14] may reflect discrepancies related to effects on the systemic inflammatory response driven by COVID-19 and the pneumonic process specifically.

It is important to appreciate that our analyses investigated associations of genetically proxied IL6R inhibition with susceptibility to COVID-19 and pneumonia, and caution should be taken when extrapolating these findings to assume the effect of a clinical intervention targeting this. Furthermore, our analysis did not directly investigate the effect of IL6R inhibition in individuals suffering with COVID-19, and therefore should not be assumed to offer insight into IL6R blockade as a target for treating COVID-19. It is similarly important to appreciate that the pneumonia analyses are likely to be dependent upon the case definition used. A further potential shortcoming is that variants proxying IL6R inhibition were only available at the IL6R locus. However, genetic associations at this locus have previously been shown to be concordant with observed effects of tocilizumab, an IL-6 blocker, supporting our claim that variants in this locus can be used as proxies for IL6R inhibition. Another limitation is that the amount of variability in CRP concentrations explained by the seven variants is small. Finally, the COVID-19 Host Genetics Initiative genome-wide association meta-analysis consists of mixed-population, which could induce bias by ancestry-difference. However, results were similar in the analysis based on the genome-wide association study comprising Italian and Spanish participants, which would be expected to be less influenced by population stratification bias.

An adverse effect of IL6R inhibition on risk of pneumonia is biologically plausible, and respiratory tract infections, including pneumonia, are a well-known complication of IL6R blockade [15]. Respiratory

\begin{tabular}{ll|rr} 
Outcome & Cases/controls & & OR (95\% CI) \\
\hline $\begin{array}{l}\text { COVID-19 } \\
\text { COVID-19 HGI }\end{array}$ & $17965 / 1370547$ & $0.97(0.94-0.99)$ \\
Hospitalised COVID-19 \\
COVID-19 HGI
\end{tabular}

FIGURE 1 Associations of genetically proxied IL6R inhibition with coronavirus disease 2019 (COVID-19) and pneumonia. Estimates were derived using the multiplicative random-effects inverse variance weighted method and accounting for the correlations between the seven genetic variants in or adjacent to the IL6R locus. \#: defined as severe respiratory confirmed COVID-19 in the COVID-19 Host Genetics Initiative (HGI) and as COVID-19 with respiratory failure in the genome-wide association study (GWAS) in Italy and Spain. CRP: C-reactive protein. 
disease is a main feature of severe COVID-19, and the potential of IL6R blockade to increase risk of pneumonia warrants vigilance and caution in their application to treat COVID-19.

Susanna C. Larsson $\circledast^{1,2}$, Stephen Burgess ${ }^{3,4}$ and Dipender Gill ${ }^{5,6,7,8}$

${ }^{1}$ Unit of Cardiovascular and Nutritional Epidemiology, Institute of Environmental Medicine, Karolinska Institutet, Stockholm, Sweden. ${ }^{2}$ Dept of Surgical Sciences, Uppsala University, Uppsala, Sweden. ${ }^{3}$ Dept of Public Health and Primary Care, University of Cambridge, Cambridge, UK. ${ }^{4}$ MRC Biostatistics Unit, University of Cambridge, Cambridge, UK. ${ }^{5}$ Dept of Epidemiology and Biostatistics, School of Public Health, St Mary's Hospital, Imperial College London, London, UK. ${ }^{6}$ Clinical Pharmacology and Therapeutics Section, Institute of Medical and Biomedical Education and Institute for Infection and Immunity, St George's, University of London, London, UK. ${ }^{7}$ Clinical Pharmacology Group, Pharmacy and Medicines Directorate, St George's University Hospitals NHS Foundation Trust, London, UK. ${ }^{8}$ Novo Nordisk Research Centre Oxford, Old Road Campus, Oxford, UK.

Correspondence: Susanna C. Larsson, Unit of Cardiovascular and Nutritional Epidemiology, Institute of Environmental Medicine, Karolinska Institutet, SE-171 77 Stockholm, Sweden. E-mail: susanna.larsson@ki.SE

Received: 17 Sept 2020 | Accepted after revision: 29 Oct 2020

Acknowledgements: The authors thank the investigators of the COVID-19 genome-wide association study, the COVID-19 Host Genetics Initiative, and FinnGen consortium for sharing summary-level data.

Conflict of interest: S.C. Larsson reports grants from Swedish Research Council for Health, Working Life and Welfare, Swedish Research Council and Swedish Heart-Lung Foundation, outside the submitted work. S. Burgess has nothing to disclose. D. Gill is employed part-time by Novo Nordisk, outside the submitted work.

Support statement: S.C. Larsson acknowledges research support from the Swedish Heart-Lung Foundation (Hjärt-Lungfonden, 20190247), the Swedish Research Council (Vetenskapsrådet, 2019-00977), and the Swedish Research Council for Health, Working Life and Welfare (Forte, 2018-00123). S. Burgess is supported by a Sir Henry Dale Fellowship jointly funded by the Wellcome Trust and the Royal Society $(204623 / \mathrm{Z} / 16 / \mathrm{Z})$. D. Gill is supported by the British Heart Foundation Research Centre of Excellence (RE/18/4/34215) at Imperial College London. This work was supported by funding from the National Institute for Health Research (Cambridge Biomedical Research Centre at the Cambridge University Hospitals National Health Service Foundation Trust). The views expressed are those of the authors and not necessarily those of the National Health Service, the National Institute for Health Research or the Department of Health and Social Care. Funding information for this article has been deposited with the Crossref Funder Registry.

\section{References}

1 Tanaka T, Narazaki M, Kishimoto T. IL-6 in inflammation, immunity, and disease. Cold Spring Harb Perspect Biol 2014; 6: a016295.

2 McElvaney OJ, McEvoy N, McElvaney OF, et al. Characterization of the inflammatory response to severe COVID-19 illness. Am J Respir Crit Care Med 2020; 202: 812-821.

3 Chen LYC, Hoiland RL, Stukas S, et al. Confronting the controversy: interleukin-6 and the COVID-19 cytokine storm syndrome. Eur Respir J 2020; 56: 2003006.

4 RECOVERY Collaborative Group, Horby P, Lim WS, et al. Dexamethasone in hospitalized patients with Covid-19 - preliminary report. N Engl J Med 2020; in press [https://doi.org/10.1056/NEJMoa2021436].

5 Lan SH, Lai CC, Huang HT, et al. Tocilizumab for severe COVID-19: a systematic review and meta-analysis. Int $J$ Antimicrob Agents 2020; 56: 106103.

6 Roche. Roche provides an update on the phase III COVACTA trial of Actemra/RoActemra in hospitalised patients with severe COVID-19 associated pneumonia. www.roche.com/media/releases/med-cor-2020-07-29.htm Date last accessed: 31 Jul 2020.

7 Coomes EA, Haghbayan H. Interleukin-6 in Covid-19: a systematic review and meta-analysis. Rev Med Virol 2020; 30: $1-9$.

8 Georgakis MK, Malik R, Gill D, et al. Interleukin-6 signaling effects on ischemic stroke and other cardiovascular outcomes: a Mendelian randomization study. Circ Genom Precis Med 2020; 13: e002872.

9 Covid-19 Host Genetics Initiative. The COVID-19 Host Genetics Initiative, a global initiative to elucidate the role of host genetic factors in susceptibility and severity of the SARS-CoV-2 virus pandemic. Eur J Hum Genet 2020; 28: 715-718.

10 Ellinghaus D, Degenhardt F, Bujanda L, et al. Genomewide association study of severe Covid-19 with respiratory failure. N Engl J Med 2020; 383: 1522-1534.

11 FinnGen consortium. FinnGen Documentation of R3 release, 2020. https://finngen.gitbook.io/documentation/ Date last accessed: 8 Oct 2020.

12 Hemani G, Zheng J, Elsworth B, et al. The MR-Base platform supports systematic causal inference across the human phenome. Elife 2018; 7: e34408.

13 Yavorska OO, Burgess S. MendelianRandomization: an R package for performing Mendelian randomization analyses using summarized data. Int J Epidemiol 2017; 46: 1734-1739.

14 Stone JH, Frigault MJ, Serling-Boyd NJ, et al. Efficacy of tocilizumab in patients hospitalized with Covid-19. N Engl J Med 2020; 383: 2333-2344.

15 Smolen JS, Schoels MM, Nishimoto N, et al. Consensus statement on blocking the effects of interleukin-6 and in particular by interleukin-6 receptor inhibition in rheumatoid arthritis and other inflammatory conditions. Ann Rheum Dis 2013; 72: 482-492. 